(Aus dem physiologischen Institut zu Breslau.)

\title{
Ueber die Abfuhrwege des Zuckers aus dem Dünndarm.
}

\author{
Von
}

Siegmund Ginsberg,

Cand. med.

Vor 11 Jahren hat v. Mering ${ }^{1}$ ) in dem physiologischen Institute zu Leipzig den Nachweis gefiuhrt, dass der Zuckergehalt des aus dem Ductus thoracicus von Hunden gewonnenen Chylus von ihrer Ernährungsweise unabhängig ist: er war bei Einführung. erheblicher Zuckermengen in die Verdauungswege nicht grösser, als bei Fleischfütterung oder im Hungerzustande, während der Zuckergehalt des Pfortaderblutes bei zuckerreicher Nahrung ohne Zweifel in die Höhe ging ${ }^{2}$ ). v. Mering folgerte daraus mit Recht, dass die Chylus-Gefässe keinen wesentlichen Antheil an der Resorption des Zuckers aus der Darmhöhle nähmen. Eine Vermuthung, aus welchen Ursachen der Zucker ausschliesslich den Blutcapillaren der Darmschleimhaut als Beute anheimfalle, hat v. Mering nicht geäussert.

In seinen kürzlich veröffentlichten „Beiträgen zur Histologie u. Physiologie der Dünndarmschleimhant" hat R. He idenhain ${ }^{3}$ ), gestiitzt auf anatomische und physiologische Thatsachen, einen Grund für jenes Verhalten nahegelegt. Er fand, dass das Wasser aus dem Dünndarm, so lange dasselbe nur in den geringen Mengen vorhanden ist, welche man unter normalen Verbältnissen an-

1) v. Mering, Ueber die Abzugswege des Zuckers aus der Darmhöhle. Arch. f. Anat. u. Physiol. Physiol. Abtheilung 1877, S. 379.

2) Vgl. auch Bleile, Ueber den Zuckergehalt des Blutes, Archiv für Anat. u. Physiol. Physiol. Abtheilung 1879, S. 59.

3) R. Heidenhain, Beiträge zur Histologie u. Physiologie der Dünndarmschleimhaut. Pflüger's Archiv 1888, Supplementband S. 71. 
trifft, ausschliesslich durch die Blutcapillaren aufgenommen wird; diese liegen dicht unter der Epithelschicht, kommen also zuerst mit der durch das Epithel in sehr langsamem Strome (vgl. a. a. O. S. 60) dringenden Flüssigkeit in Berïhrung. Die Flüssigkeit kann zu dem central gelegenen Lymphraume nicht gelangen, weil sie bereits durch das periphere Capillarnetz entführt wird. Die Wege des Wassers aber werden wohl auch die in demselben gelösten Substanzen einschlagen. Bei den Versuchen Heidenhain's hat sich aber weiter herausgestellt, dass bei Einführung ungewöhnlich grosser Flüssigkeitsmengen in den Dünndarm allerdings auch von den Chylus-Gefässen Wasser aufgenommen wird, wenn schon in weit geringerer absoluter Menge als von den Blutgefässen.

Demgemäss liess sich erwarten, dass unter diesen Umständen mit dem Wasser auch in ihm gelöste Substanzen, z. B. Zucker, in den Chylus gelangen würden. Mit Bezug auf die hier und da auftretende Neigung, in der Vertheilung der resorbirbaren Nährstoffe auf die Blut- und Lymph-Gefässe den Ausdruck einer Art von Wahl- bezw. Ausschliessungsvermögen der die Wandung dieser Canäle zusammensetzenden Zellen zu sehen, schien es erwünscht, die Haltbarkeit jener Auffassung Heidenhain's unmittelbar zu prïfen, nach welcher die Resorption des Zuckers ausschliesslich dureh die Blutgefässe Folge einerseits der anatomischen Anordnung der Gefässe in den Zotten, andrerseits der Anwesenheit nur geringer Flüssigkeitsmengen im Dünndarme ist, welche in langsamem Strome zu den Blutcapillaren durch das Epithel gelangen, so dass diese sie vollständig in Beschlag nehmen können.

Prof. Heidenhain forderte mich desshalb auf, Versuche daruber anzustellen, ob eine Vermehrung des Zuckergehaltes im Chylus nachweisbar sei, wenn mit dem Zueker in die Verdaunngswege so grosse Flüssigkeitsmengen eingeführt werden, dass die Blutcapillaren derselben nicht mehr Herr zu werden vermögen.

In dem Folgenden berichte iqh über die Resultate.

I.

\section{Versuche an Kaninchen.}

1. Method e.

a) Fistel des Ductus thoracicus.

Gewonnen wurde der Chylus aus einer Fistel des Ductus thoracicus, deren Anlegung eine nicht ganz leichte Operation ist. Sie 
wurde stets von Prof. Heidenhain ausgeführt. Als verlässlichste Methode hat sich folgende herausgestellt. Der Ductus thoracicus ist in ungefülltem Zustande schwer auffindbar: es ist deshalb zweckmässig, denselben zu starker Anfüllung zu zwingen. Man erreicht dies, indem man der Reihe nach die V.V. jugularis interna, jugularis externa, subclavia auf der linken Seite, nahe ibrem Vereinigungswinkel unterbindet, alle ihre Nebenäste schljesst und zuletzt auch noch die Vena cava superior sinistra ligirt. Kaum ist das Letztere geschehen und dem Chylus damit der letzte Ausweg abgesperrt, so springt der Ductus thoracicus stark gefïllt hervor. Er mündet nicht immer als einfacher Stamm, sondern oft mehrfach gespalten in eine dreieckige Tasche, in welche von der Kopfseite her ein längs der Vena jugularis externa herabsteigender Lymphstamm von ziemlicher Weite sich einsenkt, der ebenfalls so weit anschwillt, dass er leicht unterbunden werden kann. Jene Tasche, welche eine Art sinus lymphaticus darstellt, der den Chylus in die Vene überführt, gestattet mitunter unmittelbar die Einführung einer Glas-Canile; in andern Fällen macht ibre geringe Breite es nothwendig, die Cantile in den oben erwähnten, vom Kopfe her kommenden Lymphstamm einzulegen, die Mundung derselben natürlich gegen den Sinus gerichtet. Auf diese Weise kann man den Chylus leicht sammeln. Gegenüber den Versuchen an Hunden baben solche an Kaninchen den Vorzug, dass der Chylus nur selten gerinnt. Um ein regelmässiges $A$ bströmen herbeizufuhren, empfiehlt es sich, das (auf dem Rïcken fixirte) Kaninchen in ein Handtuch zu wickeln und dieses ein wenig angezogen zu fixiren. Eine ganz wesentliche Beschleunigung erhält ja bekanntlich der Strom des Chylus durch die A thembewegungen, weil bei jeder Einathmung der Druck in der Bauchhöhle steigt. Die Bauchwandungen des Kaninchens sind aber ausserordentlich nachgiebig, so dass das herabsteigende Zwerchfell auf die Därme einen nur geringen Druck aus zuiiben vermag, so lange nicht der Widerstand der Bauchwandungen von aussen ber durch eine mässig feste Einwicklung unterstïtzt wird. Die ersten Tropfen des aus der Canüle fliessenden Chylus liessen wir in der Regel unbenutzt, weil sich ihnen häufig eine Spur Blut beigemischt hatte, das bei der Unterbindung der Venen in der oben erwähnten Tasche zurückgeblieben war. Den folgenden Chylus aber fingen wir, um seine Gerinnung zu verhindern, in einer kalt gesättigten Lösung von schwefelsaurem Na- 
tirum auf, gewöhnlich so, dass wir in einen $10 \mathrm{ccm}$-Maasscylinder $3 \mathrm{~cm}$ Natriumsulphat einliessen und die $7 \mathrm{~cm}$ Chylus alsdann durch Umschiitteln mit dem Natriumsulphat zur Vermischung brachten. Während des Auffangens wurde, soweit dies nicht durch Gerinnungen oder andere Zufälle beeinträchtigt ward, die in bestimmten Zeiten ausgeflossene Menge Chylus notirt. Bis zum nächsten Tage, wo erst die chemische Verarbeitung vorgenommen werden konnte, wurde der Chylus, ebenso auch das Blut, im Eisschrank aufbewahrt. Letzteres nahmen wir aus der Carotis und fingen es gleichfalls in schwefelsaurem Natrium auf. Vor dem Versuche bekamen die Thiere Morphium, während des Versuches, so oft es erforderlich war, Chloroform und Aether zu gleichen Theilen gemischt. Bei dieser Art der Narkose treten bäufig Aenderungen der Zahl und Tiefe der Athemzïge ein: beide wachsen, wenn die Narkose nachzulassen beginnt, und nehmen wieder ab, wenn bei erneutem Chloroformiren die Narkose wieder tiefer wird. Entsprechend den Aenderungen der Respiration aber ändert sich die Geschwindigkeit der Entleerung des Chylus in weiten Grenzen, so dass seine Ausflussgeschwindigkeit kein Maass für seine Bildung sein kann, wie dies bei curarisirten Thieren and regelmässiger künstlicher Athmung der Fall ist.

\section{b) Chemische Untersuchung.}

Die chemischen Untersuchungen habe ich in dem chemischen Laboratorio des Instituts unter steter Leitung des Herrn Dr. Röhmann angestellt.

Verwandt zur Analyse wurden beim Kaninchen an Chylus durehschnittlich $15-20 \mathrm{ecm}$, an Blut $25 \mathrm{ccm}$. Zunächst handelte es sich darum, sowohl aus dem Chylus wie aus dem Blute das Eiweiss zu entfernen. Zu diesem Behufe ward der Chylus mit 1/10 Normal-Schwefelsäure genau neutralisirt, das Blut mit verdünnter Essigsäure (auf $25 \mathrm{cem}$ Blut etwa 2,5 ccm einer auf das Zwanzigfache verdünnten officinellen Essigsäure) versetzt, und beide auf dem Wasserbade bis zur möglichst vollkommenen Ausscheidung das Eiweisses gekocht. Um die geringen Mengen der nicht ausgefallenen Eiweisskörper vollkommen zu entfernen, wurde zu dem coagulirten Chylus, ohne zu filtriren, Salzsäure und Phosphorwolframsäure gesetzt, filtrirt, mit Natronlauge alkalisirt und auf 
ein bestimmtes Volumen aufgefiillt. War durch das Auswaschen des Niederschlages die Flüssigkeitsmenge im Verhältniss zu der Menge des zu erwartenden Zuckers zu gross geworden, so wurde in essigsaurer Lösung eingedampft und vor dem Auffüllen wieder alkalisirt. Analog wurde beim Blut verfahren, nur wurde bier schon einmal vor der Fällung mit Phosphorwolframsäure filtrirt und das Blut-Coagulum gnt ausgewaschen. Der Zucker wurde mit Fehling'scher Lösung unter Berücksichtigung der Soxhlet'schen Principien in folgender Weise titrirt. Zunächst wurde mittelst einer $0,1 \%$ igen Lösung von reinem, wiederholt ans Methylalkohol umerystallisirtem Traubenzucker der Wirkungswerth eines aus einer engen Bürette abfliessenden Cubikcentimeters Fehling'seher Lösung genau bestimmt. Die Zuckerlösung wurde auf einmal zur Fehling'schen Lösung aus einer Bürette zugesetzt, und darauf kurze Zeit im Reagenzglase zum Sieden erhitzt. Nach Absetzen des Kupferoxyduls wurde die Färbung der Fliissigkeit beobachtet. War noch ein blaner Schimmer vorhanden, was man bei Betrachtung gegen einen weissen Hintergrund nach einiger Uebung leicht entscheiden konnte, so wurde eine neue Probe gemacht, bei der etwas mehr Zuckerlösung hinzugesetzt ward, und so fortgefahren, bis die Titrirung auf $1 / 10 \mathrm{ccm}$ genau war. Sollte jetzt der Zuckergehalt, z. B. im Chylus, bestimmt werden, so wurde von der genan gemessenen, vollkommen eiweissfreien Flissigkeit zuerst in einer Vorprobe annähernd das Reduktionsvermögen für 1 ccm Fehling'scher Lösung bestimmt, dann soweit verdünnt, dass zur Reduktion $2-5 \mathrm{ccm}$ erforderlich waren. Waren weniger als $5 \mathrm{cem}$ erforderlich, so wurden aus einer Bürette so viel Cubikcentimeter destillirtes Wasser zugesetzt, dass Zuckerlösung plus Wasser plus $1 \mathrm{ecm}$ Fehling'scher Lösung $=6 \mathrm{ccm}$ war. Im Uebrigen wurde genau so wie bei der Titre-Stellung verfahren. In den meisten Fällen wurde beim Chylus die zu titrirende Fliissigkeit auf das Doppelte des ursprünglichen Chylus-Volumens verdünnt. Beim Blute musste in vielen Fällen nur auf das gleiche Volumen aufgefüllt und, in analoger Weise wie sonst mit $1 \mathrm{cem}$, so hier mit $1 / 2 \mathrm{ccm}$ Fehling tritirt werden.

Die bei der Mehrzahl der Versuche mitgelieferten Trockenbestimmungen wurden in der Weise ausgeführt, dass eine kleine Menge Chylus in einem Porzellantiegelchen, eine kleine Menge Blut in einem Glasschälchen, das dann mit einem zweiten etwas 
grösseren bedeckt wurde, aufgefangen und gewogen ward; Tiegelchen wie Schälchen wurden nun zunächst bei circa $80^{\circ} \mathrm{C}$., später bei $110^{\circ}$ bis zu constantem Gewichte getrocknet.

\section{Resultate.}

a) Bei Thieren mit normaler Fütterung (Mohrriben, Salat).

Darüber stehen uns 6 Versuche zur Verfügung, deren Ergebnisse wir in der nachfolgenden Tabelle I mittheilen: 5 Versuche beziehen sich auf Kaninchen, die längere Zeit mit Mohrrüben gefiittert worden waren, der letate auf ein Salat-Kaninchen.

Tabelle I.

\begin{tabular}{|c|c|c|c|c|c|}
\hline \multirow{2}{*}{ No. } & \multirow{2}{*}{$\begin{array}{c}\text { Gewicht } \\
\text { in } \\
\text { Kgr. }\end{array}$} & \multicolumn{2}{|c|}{ Blut } & \multicolumn{2}{|c|}{$\mathrm{Chylus}$} \\
\hline & & $\begin{array}{c}\text { Zucker- } \\
\text { Gehalt }\end{array}$ & $\begin{array}{c}\text { Trocken- } \\
\text { Gehalt }\end{array}$ & $\begin{array}{c}\text { Zucker- } \\
\text { Gehalt }\end{array}$ & $\begin{array}{c}\text { Trocken- } \\
\text { Gehalt }\end{array}$ \\
\hline 1. & $21 / 2$ & - & - & $0,25 \%$ & - \\
\hline 2. & $23 / 4$ & $0,18 \%$ & - & $0,23 \%$ & - \\
\hline 3. & $21 / 2$ & $0,18 \%$ & - & $0,25 \%$ & - \\
\hline 4. & 3 & $0,18^{\circ} \%$ & $18 \%$ & $0,23 \%$ & $4 \%$ \\
\hline 5. & $21 / 4$ & $0,18 \%$ & $16 \%$ & $0,23 \%$ & $5 \%$ \\
\hline 6. & $21 / 2$ & $0,14 \%$ & $13,6 \%$ & $0,23 \%$ & $4 \%$ \\
\hline Mittel: & - & $0,17 \%$ & $15,9 \%$ & $0,237 \%$ & $4,3 \%$ \\
\hline
\end{tabular}

In der obigen Tabelle fällt die grosse Constanz des Zuckergehaltes sowohl im Blute, wie im Chylus auf. Bestätigt wird durch die Zahlen die schon früher gemachte Erfahrung, dass der Zuckergehalt des Chylus grösser ist. als der des Blutes ${ }^{1}$ ): das Verhältniss der Mittelwerthe ist 1,39: 1. Eine Bestimmung des Zuckergehaltes im Blut-Serum war nicht durchführbar. Nimmt man an, dass $100 \mathrm{Vol}$. Blut in runder Ziffer $40 \mathrm{Vol}$. Körperchen und $60 \mathrm{Vol}$.

1) Vgl. v. Gorup-Besanez, physiol. Chemie. Braunschweig 1862, S. 360 . 
Plasma enthalten, so würde sich der Zuckergehalt in letzterem, die Körperchen als zuckerfrei vorausgesetzt, zu 0,28\% berechnen, also grö̈sser sein, als im Chylus.

b) Bei Thieren nach Zucker-Injektion.

Die Traubenzucker-Lösung erhielt das Kaninchen vor der Operation durch die Schlundsonde. Erst eine Stunde später wurde das Thier narkotisirt, so dass die Resorption zur Zeit der Operation bereits im Gange war. Vier derartige Versuche stellten wir an, ïber deren Resultate siehe:

Tabelle II.

\begin{tabular}{|c|c|c|c|c|c|c|}
\hline \multirow[b]{2}{*}{ No. } & \multirow{2}{*}{$\begin{array}{c}\text { Gewicht } \\
\text { in } \\
\text { Kgr. }\end{array}$} & \multicolumn{2}{|c|}{ Blut } & \multicolumn{2}{|c|}{ Chylus } & \multirow[b]{2}{*}{ Injicirt: } \\
\hline & & $\begin{array}{c}\text { Zucker- } \\
\text { Gehalt }\end{array}$ & $\begin{array}{c}\text { Trocken- } \\
\text { Gehalt }\end{array}$ & $\begin{array}{c}\text { Zucker- } \\
\text { Gehalt }\end{array}$ & $\begin{array}{c}\text { Trocken- } \\
\text { Gehalt }\end{array}$ & \\
\hline 1. & $21 / 2$ & $0,22 \%$ & - & $0,36 \%$ & - & $\begin{array}{l}50 \mathrm{ccm} \text { Wasser mit } 5 \mathrm{gr} \\
\text { Zucker. Respiration } \\
\text { furchtbar träg. }\end{array}$ \\
\hline 2. & 2 & $0,39 \%$ & $13,5 \%$ & $0,76 \%$ & $4^{0} \%$ & $\begin{array}{l}100 \mathrm{ccm} \text { Wasser mit } \\
15 \mathrm{gr} \text { Zucker. }\end{array}$ \\
\hline 3. & $2^{1} / 2$ & $0,33 \%$ & $18 \%$ & $0,39 \%$ & $4 \%$ & $\begin{array}{l}100 \mathrm{ccm} \text { Wasser mit } \\
15 \mathrm{gr} \text { Zucker. }\end{array}$ \\
\hline 4. & 3 & $0,31 \%$ & $17 \%$ & $0,46 \%$ & $5 \%$ & $\begin{array}{l}150 \mathrm{ccm} \text { Wasser mit } \\
25 \mathrm{gr} \text { Zucker. }\end{array}$ \\
\hline Mittel & - & $0,31 \%$ & $15,5 \%$ & $0,49 \%$ & $4,3 \%$ & \\
\hline
\end{tabular}

Die Zucker-Injektion bat also ein Steigen des Zuckergehaltes sowohl des Blutes wie des Cbylus zur Folge, und es scheint sich demnach, zunächst für das Kaninchen, die von Heidenhain ausgesprochene Vermuthung zu bestätigen, dass der Zuckergehalt auch des Chylus steige, wenn in die Verdaunngswege Zucker zugleich mit viel Wasser eingeführt werde.

Die Schlüsse aus den Beobachtungen am Kaninchen sind desshalb vielleicht anfechtbar, weil der Chylus ohne und mit Zuckereinführung nicht ein. und demselben Thiere entnommen wurde. Um an demselben Individuum den Zuckergehalt des Chylus obne und mit Einführung von Zuckerlösung in die Verdauungswege zu bestimmen, mussten wir uns an Hunde wenden. 
II.

\section{Versuche an Houden.}

1. Methode.

Die Anlegung einer Fistel des Ductus thoracieus beim Hunde ist so oft besprochen, dass die Operationsmethode als bekannt vorausgesetzt werden darf. Nachdem die Canüle in den Ductus thoracicus eingelegt war, wurde bei dem (stets vollständig und während der ganzen Versuchsdauer durch Morphium und Chloroform narkotisirten) Thiere die Bauchhöble unterhalb des Nabels auf eine kurze Strecke in der Linea alba eröffnet und die Einmündungsstelle des Dünndarms in den Dickdarm aufgesucht, um in jenen eine aufwärts gerichtete Canüle behufs späterer Einführung der Zuckerlösung einzulegen. War dies geschehen, so wurde die Bauchhöhle wieder geschlossen und nur die Canïle nach aussen geleitet, versehen mit einem Gummischlauche, an dessen anderm Ende sich ein Glastrichter befand. Gerade diese Operation vereitelte resp. beeinträchtigte einen oder den andern Versuch: denn beim Hervorsuchen des Dünndarmendes durch die kleine Bauchwunde ging es hier und da nicht ohne Schädigung des Darmes ab, infolge wovon es zu kleinen Blutungen in der Darmwand kam, die ihrerseits wieder ein Blutigwerden des Chylus und ein fast ununterbrochenes Gerinnen desselben hervorriefen. Vorlaufig ward noch kein Zucker in den Darm injicirt; dies geschah erst, nachdem eine genügende Menge Chylus sowie Blut (letzteres, um in der Nachbarschaft der Ductus-Caniile keinerlei Störung zu machen, der Cruralis entnommen) zur Zucker- und Trocken-Bestimmung genommen war. Danach ward also die körperwarme ZuckerLösung in den Darm injicirt, gewöhnlich zwei Mal $200 \mathrm{ccm}$, zwischen beiden Injektionen lag etwa eine Stunde; wieder ward von der ersten Zuckerinjektion ab Chylus und am Ende des Versuches Blut zur Zucker- wie zur Trocken-Bestimmung anfgefangen. Nach Tödtung des Versuchsthieres wurde in Darm und Magen nachgesehen, wie viel Flüssigkeit sich vorfände, um zu wissen, ob die Resorption in erwünschter Weise vor sich gegangen wäre; der meist ganz beträchtliche Rest war nicht bloss Zuckerlösung, sondern auch Darm- und Magensaft, deren Sekretion infolge der ziemlichen Concentration der injicirten Lösungen in gesteigertem Maasse vor sich ging. 
Die chemische Verarbeitung wich in nichts von der beim Kaninchen ab. Zur Verwendung kamen durchsehnittlich je 20 cem Chylus und je 40 gr Blut vor und nach der Injektion.

\section{Resultate.}

a) Einfache Traubenzucker-Injektions-Versuche.

Deren Resultate veranschaulicht die nachstehende Tabelle III. $\mathrm{Zu}$ dem ersten der Versuche muss bemerkt werden, dass er völlig negativ ausfiel: nach der Zuckerinjektion beschleunigte sich der Chylus-Ausfluss nicht, sondern verringerte sich sogar, die Zusammensetzung des Blutes und des Chylus änderte sich nicht; es hatte also aus unbekannten Gründen keine Resorption stattgefunden. Gefiittert waren die Thiere sämmtlich reichlich mit fettem Fleisch am Abend vor dem Versuche.

Tabelle III.

\begin{tabular}{|c|c|c|c|c|c|c|c|c|c|c|}
\hline \multirow[b]{2}{*}{ No. } & \multirow{2}{*}{ 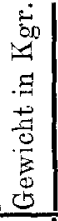 } & \multicolumn{2}{|c|}{$\begin{array}{l}\text { Blut vor } \\
\text { Injektion }\end{array}$} & \multicolumn{2}{|c|}{$\begin{array}{l}\text { Blut nach } \\
\text { Injektion }\end{array}$} & \multicolumn{2}{|c|}{$\begin{array}{l}\text { Chylus vor } \\
\text { Injektion }\end{array}$} & \multicolumn{2}{|c|}{$\begin{array}{c}\text { Chylus nach } \\
\text { Injektion }\end{array}$} & \multirow[b]{2}{*}{ Injicirt: } \\
\hline & & 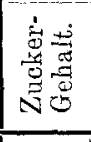 & 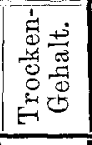 & 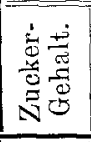 & 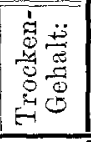 & 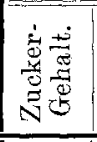 & 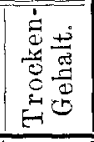 & 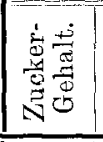 & 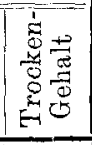 & \\
\hline 2. & 12 & $\begin{array}{l}0,14 \% \\
0,07 \% \\
0,09 \% \\
0,08 \%\end{array}$ & $\begin{array}{l}23,0^{\circ} \% \\
19,8^{0} \% \\
22,0 \%\end{array}$ & $\begin{array}{l}0,14 \% \\
0,27 \% \\
0,18 \% \\
0,28 \%\end{array}$ & $\begin{array}{l}20,0 \% \\
18,9 \% \\
21,0 \%\end{array}$ & $\left|\begin{array}{l}0,24 \% \\
0,25 \% \\
0,22 \% \\
0,16 \%\end{array}\right|$ & $\left|\begin{array}{c}14,0 \% \\
- \\
5,8 \% \\
9,0 \%\end{array}\right|$ & $\left|\begin{array}{l}0,27 \% \\
0,52 \% \\
0,35 \% \\
0,42 \%\end{array}\right|$ & $\begin{array}{c}9,0 \% \\
- \\
5,6 \% \\
5,0 \%\end{array}$ & $\begin{array}{l}600 \mathrm{ccm} \text { Wasser } \\
\text { m. 30gr Zucker. } \\
400 \mathrm{ccm} \text { Wasser } \\
\text { m. } 40 \mathrm{gr} \text { Zucker. } \\
4.00 \mathrm{~cm} \text { Wasser } \\
\text { m. } 20 \mathrm{gr} \text { Zucker. } \\
400 \mathrm{ccm} \text { Wasser } \\
\text { m.30gr Zucker. }\end{array}$ \\
\hline suchir 3.4. & - & $0,08 \%$ & $1,6 \%$ & $0,24 \%$ & $20 \%$ & $0,21 \%$ & $7,4 \%$ & $0,43 \%$ & $5,3 \%$ & \\
\hline
\end{tabular}

Nach Ausweis der drei gelungenen Versuche hat also beim Hunde die Einführung von Zucker zugleich mit viel Flüssigkeit ein Steigen des Zuckergehaltes sowohl im Blute wie im Chylus zur Folge.

b) Kochsalz-Injektions-Versuche.

Angestellt wurden dieselben, um dem etwaigen Einwande zu begegnen, als wäre es nicht der Zucker, der jene Steigerung des Zuckergehaltes hervorriefe, sondern die blosse Resorption einer 
grossen Flüssigkeitsmenge, vielleicht indem dieselbe Zucker aus der Leber oder andern Geweben heraus- und in die Lymphe und den Chylus hineinspüle. Die Versuchsanordnung blieb dieselbe, nur wurde eben statt Zucker eine Kochsalzlösung in den Darm injicirt, und zwar in beiden Versuchen $400 \mathrm{cem}$ einer $0,3 \%$ igen Kochsalzlösung. Folgendes war das Resultat der beiden Versuche:

Tabelle IV.

\begin{tabular}{|c|c|c|c|c|c|c|c|c|c|}
\hline \multirow[b]{2}{*}{ 完 } & \multirow{2}{*}{$\begin{array}{c}\text { Ge- } \\
\text { wicht } \\
\text { in } \\
\text { Kgr. }\end{array}$} & \multicolumn{2}{|c|}{$\begin{array}{l}\text { Blut vor } \\
\text { Injektion }\end{array}$} & \multicolumn{2}{|c|}{$\begin{array}{l}\text { Blut nach } \\
\text { Injelstion }\end{array}$} & \multicolumn{2}{|c|}{$\begin{array}{l}\text { Chylus vor } \\
\text { Injektion }\end{array}$} & \multicolumn{2}{|c|}{$\begin{array}{c}\text { Chylus nach } \\
\text { Injektion }\end{array}$} \\
\hline & & 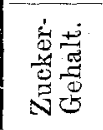 & 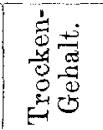 & 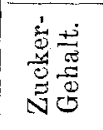 & 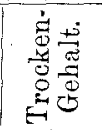 & 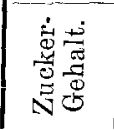 & 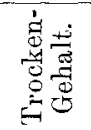 & 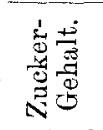 & 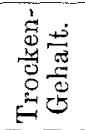 \\
\hline 1. & 16 & $0,04 \%$ & $23,0 \%$ & $0,03 \%$ & $22,0 \%$ & $0,09 \%$ & $7,9 \%$ & $0,10 \%$ & $3,9 \%$ \\
\hline 2. & 15 & $0,06 \%$ & $19,3 \%$ & $0,06 \%$ & $18,4 \%$ & - & $7,6 \%$ & - & $6,0^{\circ} \%$ \\
\hline
\end{tabular}

Es zeigt sich also, dass die Resorption grosser Flussigkeitsmengen für sich keine wesentlichen Aenderungen des Zuckergehaltes, weder im Blute noch im Chylus hervorruft.

Die obigen Versuche erfüllen die von Heidenhain ausgesprochene Erwartung, dass unter passenden Bedingungen Zucker aus dem Darme nicht bloss in die Blut-, sondern auch in die Chylus-Bahnen ibergehen werde, dann nämlich, wenn gleichzeitig so reichliche Wassermengen zur Aufnahme gelangen, dass die in den Zotten subepithelial gelagerten Blutcapillaren nicht die ganze durch das Epithel ihnen zugeführte Fliussigkeitsmenge aufzunehmen vermögen, sondern einen in Betracht kommenden Theil derselben an sich vorüber in die Tiefe der Zotte zu den Chylusräumen gelangen lassen: die Chylusgefässe schliessen den Zucker nicht aus, sondern nehmen ihn, wenn er ihnen geboten wird - nur dass unter gewöhnlichen Umständen, d. h. bei Anwesenheit nur kleiner Flissigkeitsmengen im Darme, eben keine Zuckerlösung bis zu ihnen gelangt.

Bei der principiellen Tragweite dieses aus den Versuchen gezogenen Schlusses schien es geboten, denselben gegen mögliche Einwände sicher zu stellen.

Man könnte vielleicht einwenden, dass der Zucker in den 
Chylus nicht unmittelbar aurs dem Darmkanale gelange, sondern zunächst in das Blut iubergeführt, in dem Körper auf die Blutbahnen vertheilt und durch diese erst der Lymphe zugeführt werde.

Angesichts der Versuche von v. Mering scheint dieser Einwand freilich von vornherein hinfällig. Als dieser Forscher Hunde mit grossen Mengen unge lösten Zuckers fütterte, sah er den Zuckergehalt des Carotiden-Blutes (es wurde der des Serums bestimmt, aus welchem sich der des Blutes schätzungsweise berechnen lässt) ungefähr so weit steigen, wie wir in den obigen Versuchen; trotzdem sah er unter ähnlichen Bedingungen den Gehalt des Chylus an Zucker sich nicht wesentlich ändern (gegenüber dem Gehalte bei hungernden oder mit Fleisch gefiitterten Thieren). Wäre in unsern Versuchen nur die Bereicherung des Blutes an Zucker maassgebend für seine Vermehrung im Chylus, so hätte v. Mering Aehnliches beobachten müssen, wie wir.

Die Bestimmung des Zuckergehaltes im Blute und im Chylus ist von v. Mering, wie es scheint, nicht bei demselben Individuum ausgeführt worden, was seinen Thatsachen eine noch grössere Sicherheit geben würde. Im Hinblick anf diesen Umstand wollten wir den obigen Einwand durch eigene Versuche prüfen, die von folgendem Gedankengange ausgingen. Wenn die Lymphe nicht direkt aus dem Darme ihren Zucker empfängt, sondern nur durch das Blut zugeführt erhält, so wird der Zuckergehalt derselben geringer ausfallen, wenn ein grösserer Theil des Kreislaufes nach Einfuihrung des Zuckers in den Darm ausgeschaltet wird. Letzteres zu bewerkstelligen, führten wir nach einer in dem Leipziger Laboratorio vielfach geübten Methode von der Art. cruralis aus einen Gummi-Obturator (aufblasbaren Ballon) in die Aorta bis uiber die Theilungsstelle derselben, so dass die Circulation in dem Becken und den hinteren Extremitäten völlig unterdrückt wurde. Es zeigte sich aber, dass nach der Zucker-Injektion in den Darm der Zuckergehalt des Chylus ebenso hoch stieg, als bei vollständig freiem Kreislauf, wie folgende Zahlen lehren. (S. folg. Seite.)

Berücksichtigt man, dass unter den Verhältnissen der obigen Versuche in den Ductus thoracicus nur noch die Lymphe aus den Bancheingeweiden, den Bauch- und Brustwandungen und einer obern Extremität (der rechten) einströmte, so wird man es für schwer glaublich halten, dass der Zuckeriiberschuss im Ductus thoracicus anderswoher als aus dem Darmehylus stamme. 
Tabelle V.

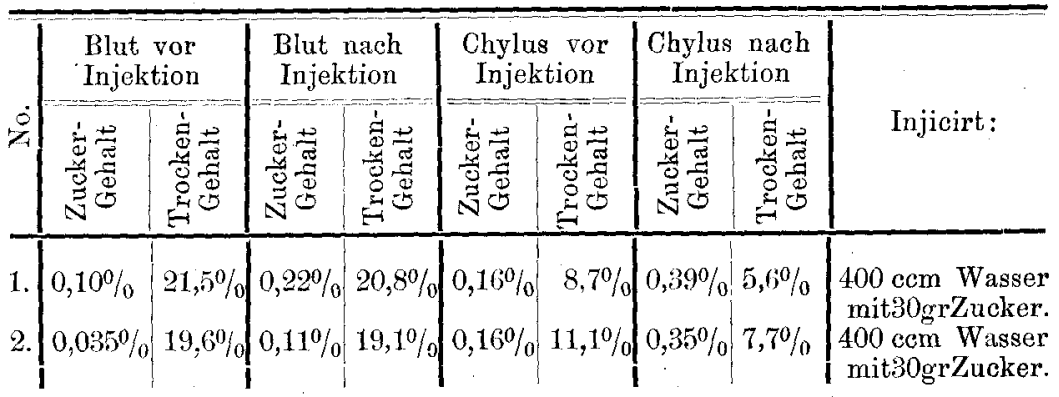

Dieser Schluss wird um so vertrauenswerther, als die Unterbrechung des Kreislaufes im Hinterkörper die Lymphmenge, welche aus dem Ductus thoracicus ausfloss, nicht wesentlich herabsetate, woraus folgt, dass auch bei freier Aorta nur sehr wenig Lymphe aus dem Becken und den binteren Extremitäten in den Milchbrustgang eingeflossen sein wird. Die Thiere lagen in tiefer Narkose ruhig da, so dass die Extremitäten-Lymphe von sehr geringem Betrage gewesen sein muss - eine Thatsache, die nach allem, was wir von den Bedingungen der Lymphbildung in den Extremitäten wissen, durchaus nicht überraschend ist.

Allenfalls könnte man gegen die letzten Versuche noch den Einwand bringen, dass nach Verschliessung der Aorta unterhalb der Nierenarterien in das Pfortadergebiet eine ungewöhnlich grosse Blutmenge hinübergedrängt und dadurch die Zuckerbildung in der Leber gesteigert worden sei: vielleicht sei so nach der Obturation eine ungewöhnlich zuckerreiche Leber-Lymphe in den Milchbrustgang geflossen. Wäre dies der Fall, so müsste eine blosse Verstopfung der Aorta ohne Zucker-Injektion in den Darm den Zuckergehalt im Ductus thoracicus in die Höhe treiben, was in Wirklichkeit nicht in merklicher Weise der Fall ist. Hierzu folgende Versucbe, bei denen eben nur die Aorta obstruirt ward. (S. f. Seite.) In dem letzten Versuche ist zwar der Zuckergehalt des Chylus nach der Obstruktion etwas gestiegen, doch ist diese Steigerung ja ganz minimal ge gen über derj c n ige $\mathrm{n}$ in den Injektions-Versuchen; die ersten drei Versuche geben einen gleichen Zuckergehalt vor und nach der Obturation. 
318 S i g mund Gins berg: Ueber die Abfuhrwege des Zuckers etc.

Tabelle VI.

\begin{tabular}{|c|c|c|c|c|c|c|c|c|}
\hline \multirow[b]{2}{*}{ मت } & \multicolumn{2}{|c|}{$\begin{array}{l}\text { Blut vor } \\
\text { Obstruktion }\end{array}$} & \multicolumn{2}{|c|}{$\begin{array}{l}\text { Blut nach } \\
\text { Obstruktion }\end{array}$} & \multicolumn{2}{|c|}{$\begin{array}{l}\text { Chylus vor } \\
\text { Obstruktion }\end{array}$} & \multicolumn{2}{|c|}{$\begin{array}{l}\text { Chylus nach } \\
\text { Obstruktion }\end{array}$} \\
\hline & 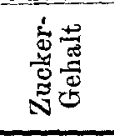 & 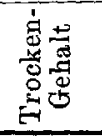 & 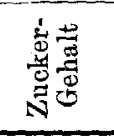 & 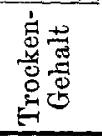 & 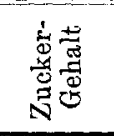 & 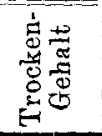 & 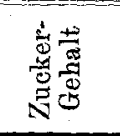 & 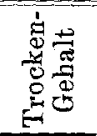 \\
\hline 1. & $0,04^{0} \%$ & $23,2 \%$ & $0,04 \%$ & $28,9 \%$ & $0,30 \%$ & $7,9 \%$ & $0,30 \%$ & $6,6 \%$ \\
\hline 4. & $0,03 \%$ & $18,8 \%$ & $0,035^{0} i_{0}$ & $18,8 \%$ & $0,105 \%$ & $12,8 \%$ & $0,14 \%$ & $12,1 \%$ \\
\hline 8 & $0,051 \%$ & $21,1 \%$ & $0,044 \%$ & $22 \%$ & $0,177 \%$ & $8,2 \%$ & $0,188 \%$ & $6,3 \%$ \\
\hline & - & - & - & - & $0,26 \%$ & $7,7 \%$ & $0,33 \%$ & $8,1 \%$ \\
\hline
\end{tabular}

Nach der Gesammtheit aller mitgetheilten Versuche ist es wohl kaum zweifelhaft, dass bei Anwesenheit reichlicher Flussigkeitsmengen im Darme gelöste Substanzen (Zucker) nicht bloss in das Blut, sondern auch in den Chylus übergehen. Wenn nach v. Mering für gewöhnlich die Blutgefässe allein den Zucker aufnebmen, so haben sie dies ibrer subepithelialen Lagerung in den Zotten zu danken: als die ersten auf dem Wege der resorbirten Fliissigkeit machen sie ibr Prioritätsrecht geltend und nehmen den Chylus-Gefässen den Zucker vorweg, so lange sie die vom Epithel her ihnen zuströmende Flüssigkeitsmenge zu bewältigen vermögen; können sie diese nicht völlig bezwingen, so kommen ihnen die Chylusgefässe bereitwilligst zu Hülfe.

Sollte Jemand trotz alledem den Chylus-Gefässen ein Ausschliessungsvermögen gegenüber dem Nahrungs-Zucker zuerkennen und der Annahme zuneigen, dass der Zuekeriberschuss des Chylus in unsern Versuchen nicht aus dem Darm unmittelbar herstamme, sondern innerhalb der Darmwand von dem an Zucker bereicherten Blute an den Chylus abgetreten sei, so wirde eine zweifellose Widerlegung schwer möglich, aber auch die Gegenfragen kaum zu beantworten sein: 1) wesshalb denn in v. Mering's Versuchen das in gleichem Grade an Zucker bereicherte Blut den Zuckergehalt des Chylus nicht gesteigert habe; 2) wesshalb in unsern Versuchen die Chylus-Gefässe zwar den aus dem Darm direkt, aber nicht ebenso den aus den Bluteapillaren stammenden Zucker ausschliessen sollten. 